\title{
Studies on NPK Biofertilizer Consortia and their Influence on Maize (Zea mays L.) Seedling Vigour
}

\author{
Ashwini $^{1^{*}}$ and C. R. Patil ${ }^{2}$ \\ ${ }^{1}$ Department of Agricultural Microbiology, College of Agriculture, \\ Dharwad, Karnataka - 580005, India \\ ${ }^{2}$ Department of Agricultural Microbiology, Institute of Organic Farming, University of \\ Agricultural Sciences, Dharwad, Karnataka - 580005, India \\ *Corresponding author
}

\section{A B S T R A C T}

\begin{tabular}{|l|}
\hline Ke y w o r d s \\
NPK consortia, \\
Gluconacetobacter, \\
Azospirillum, \\
Phosphate \\
solubilizing \\
bacterium, \\
Potassium \\
solubilizing \\
bacterium, \\
Formulations \\
\hline Article Info \\
\hline Accepted: \\
12 December 2020 \\
Available Online: \\
10 January 2021 \\
\hline \hline
\end{tabular}

Four local efficient biofertilizer strains namely, Gluconacetobacter $\mathrm{G}_{1}$ and Azospirillum ACD-15 as nitrogen fixers, Pseudomonas striata as phosphorus solubilizing bacterium and a potassium solubilizing bacterium were used in developing NPK biofertilizer consortia. Each NPK consortia consisted of one of the two nitrogen fixing bacteria, the phosphate solubilizing (PSB) and potassium solubilizing (KSB) bacteria in five different formulations namely, lignite, kaolinite, liquid, Na-alginate and bentonite. These formulations were evaluated under in vitro on maize germination and seedling vigour. Growth kinetics of each strain was used in developing consortia after their compatibility was confirmed. The population of each of the four strains in the formulations were assessed at ambient conditions over 90 days. The survival of individual strain in the formulations of consortia and their influence on maize seedling vigour were used to identify efficiency of formulations of NPK consortia. Among the five formulations, liquid and Na-alginate formulations supported significantly higher population of biofertilizer strains after 90 DAS. Liquid formulation recorded the highest maize germination (94.25 per cent) and $\mathrm{Na}$-alginate formulation recorded the highest maize seedling vigour (3850.43).

\section{Introduction}

Recent improvements in the biofertilizers has shifted the focus of modern agriculture towards environmental friendly sustainable agricultural practices which are known to reduce the dependence on chemical fertilizers and in future it is expected to offer the possibilities to reach our demand for agricultural needs (Desai et al., 2012). Biofertilizers are ready to use products consisting of live microbial inoculants which are capable of fixing atmospheric nitrogen, solubilizing phosphorus, potassium and zinc, bring about organic matter decomposition in the soil. 
Plants need macro and micronutrients for their growth and development. Individual application of each of the bioinoculants would be expensive and laborious. Hence, development of consortia with consistent efficiency under field conditions and longer shelf life will pave way for successful commercialization of this technology. Therefore, bioinoculants consisting of biofertilizer strains capable of mobilizing N, P and $\mathrm{K}$ nutrients as a single inoculant with enhanced shelf-life will be useful technology in present agriculture.

Among the soil bacteria, diazotrophs such as Gluconacetobacter and Azospirillum are found beneficial to crops which belong to graminaceous family (Raja et al., 2006). These are most commonly applied for fixing atmospheric nitrogen to enhance its availability and uptake by plants for their growth and development. In order to achieve an increased beneficial effect of these diazotrophs and other nutrient mobilizing microorganisms on growth and yield enhancement (Jain et al., 2012). Microbial consortium is a combination of more than two beneficial microorganisms and is preferred as consortial inoculation performs better than single inoculations (Alagawadi and Gaur, 1998) due to their synergistic effect in consortia.

Earlier studies conducted on the use of microbial consortia in different combinations of inoculants (Jayashree and Jagadeesh, 2017; Vijaykumar and Brahmaprakash, 2018) reported enhanced growth and yield of crops inoculated. However, studies on consortia formulated in different carriers and their effect on maize (Zea mays L.) is lacking. Hence, this investigation was conducted to develop two microbial consortia each consisting of a nitrogen fixer (Gluconacetobacter or Azospirillum), a PSB (Psuedomonas striata) and a KSB in different formulations and evaluate their effect on maize under in vitro.

\section{Materials and Methods}

The biofertilizer strains used included local efficient strains of Gluconacetobacter $\mathrm{G}_{1}$ and Azospirillum ACD-15 as nitrogen fixers, phosphorus solubilizing bacterium Psuedomonas striata and local efficient potassium solubilizing bacterium (KSB) collected from the Agricultural Microbiology Laboratory at the Institute of Organic Farming (IOF), University of Agricultural Sciences, Dharwad. All strains were purified by repeated streaking on their selective media viz., Gluconacetobacter on LGIP agar medium (Sucrose $100 \mathrm{~g} \mathrm{~L}^{-1}, \mathrm{~K}_{2} \mathrm{HO}_{4} 0.2 \mathrm{~g} \mathrm{~L}^{-1}$, $\mathrm{KH}_{2} \mathrm{PO}_{4} 0.6 \mathrm{~g} \mathrm{~L}^{-1}, \mathrm{MgSO}_{4} .7 \mathrm{H}_{2} \mathrm{O} 0.2 \mathrm{~g} \mathrm{~L}^{-1}$, $\mathrm{CaCl}_{2} .2 \mathrm{H}_{2} \mathrm{O} 0.02 \mathrm{~g} \mathrm{~L}^{-1}, \mathrm{FeSO}_{4} 0.02 \mathrm{~g} \mathrm{~L}^{-1}$, $\mathrm{Na}_{2} \mathrm{MoO}_{4} \cdot 2 \mathrm{H}_{2} \mathrm{O} 0.002 \mathrm{~g} \mathrm{~L}^{-1}, 5 \mathrm{ml}$ of $0.5 \%$ Bromothymol blue solution in $0.2 \mathrm{~N} \mathrm{KOH}$, Agar $18 \mathrm{~g} \mathrm{~L}^{-1}$ ), Azospirillum on sodium malate agar medium (Malic acid $5 \mathrm{~g} \mathrm{~L}^{-1}$, $\mathrm{KH}_{2} \mathrm{PO}_{4} 0.5 \mathrm{~g} \mathrm{~L}^{-1}, \mathrm{MgSO}_{4}$. $7 \mathrm{H}_{2} \mathrm{O} 0.2 \mathrm{~g} \mathrm{~L}^{-1}$, $\mathrm{FeSO}_{4} 0.1 \mathrm{~g} \mathrm{~L}^{-1}, \mathrm{MnSO}_{4} 0.1 \mathrm{~g} \mathrm{~L}^{-1}, \mathrm{NaCl} 0.02$ $\mathrm{g} \mathrm{L}^{-1}, \mathrm{CaCl}_{2} 0.01 \mathrm{~g} \mathrm{~L}^{-1}, \mathrm{Na}_{2} \mathrm{MoO}_{4} \cdot 7 \mathrm{H}_{2} \mathrm{O} 0.002$ $\mathrm{g} \mathrm{L}^{-1}, 2 \mathrm{ml}$ of $0.5 \%$ of Bromothymol blue solution in $0.2 \mathrm{~N} \mathrm{KOH}$, Agar $18 \mathrm{~g} \mathrm{~L}^{-1}$ ), PSB on Pikovaskaya's medium (Glucose $10 \mathrm{~g} \mathrm{~L}^{-1}$, $\mathrm{Ca}_{3}\left(\mathrm{PO}_{4}\right)_{2} \quad 5 \quad \mathrm{~g} \quad \mathrm{~L}^{-1}, \quad \mathrm{KCl} \quad 0.5 \quad \mathrm{~g} \quad \mathrm{~L}^{-1}$, $\mathrm{MgSO}_{4} .7 \mathrm{H}_{2} \mathrm{O} 0.2 \mathrm{~g} \mathrm{~L}^{-1}, \mathrm{FeSO}_{4} 0.02 \mathrm{~g} \mathrm{~L}^{-1}$, yeast extract $0.02 \mathrm{~g} \mathrm{~L}^{-1}$, Agar $18 \mathrm{~g} \mathrm{~L}^{-1}$ ) and KSB on Alekshandrov's medium (Glucose 5 $\mathrm{g} \mathrm{L}{ }^{-1}, \mathrm{FeCl}_{3} 0.1 \mathrm{~g} \mathrm{~L}{ }^{-1}, \mathrm{CaCO}_{3} 2 \mathrm{~g} \mathrm{~L}^{-1}$, potassium mineral, mica $2 \mathrm{~g} \mathrm{~L}^{-1}, \mathrm{CaSO}_{4} 2 \mathrm{~g} \mathrm{~L}^{-}$ ${ }_{1}^{1}, \mathrm{MgSO}_{4} .7 \mathrm{H}_{2} \mathrm{O} 0.005 \mathrm{~g} \mathrm{~L}^{-1}$, Agar $18 \mathrm{~g} \mathrm{~L}^{-1}$ ).

\section{Compatibility test}

Before development of consortia, all biofertilizer strains were tested for their compatibility on nutrient agar medium by cross streak method described by Anandraj and Leema (2010). 


\section{Growth studies of biofertilizer strains}

The growth of four biofertilizer strains were examined in $100 \mathrm{ml}$ broth cultures of respective selective broth media described before. The broths used were amended with cell protectants as described in Table 2 and media without cell protectants as to determine the time taken by each biofertilizer strain to reach its highest population.

\section{Composition of NPK consortia}

Two microbial consortia namely, microbial consortium-1 (MC-1) containing Gluconacetobacter $\quad \mathrm{G}_{1}+\mathrm{PSB}+\mathrm{KSB} \quad$ and microbial consortium-2 (MC-2) containing Azospirillum ACD-15+PSB+KSB were developed.

\section{Preparation of biofertilizer consortia}

Based on the equal populations of microbial strains used, two different microbial consortia consisting of one of the two nitrogen fixers (Gluconacetobacter $\mathrm{G}_{1}$ or Azospirillum ACD15), a PSB (Psuedomonas striata) and a KSB were prepared and formulated as lignite, kaolinite, bentonite, Na-alginate and liquid biofertilizer. The microbial strains were grown on respective selective medium and the two consortia were prepared by mixing them in equal proportion $(1: 1: 1)$ with $10^{8} \mathrm{CFU} / \mathrm{ml}$ (Table 3) and then, they were mixed with sterilized carrier materials (viz., lignite, kaolinite, bentonite) in the ratio 3 parts of carrier and 1 par of broth and stored in High Density Poly Ethylene (HDPE) bags of 75 gauge and liquid and Na-alginate formulations were stored in High Density Poly Ethylene (HDPE) narrow mouth bottles (Tarsons) for three months under ambient conditions for shelf life studies. By following this method the differences that could have arisen due to variations in their populations and differential growth rate were avoided. To achieve this, the strains were inoculated in a staggered system so as to get their maximum populations coinciding with the time of formulating consortia.

Liquid formulation technology of Gluconacetobacter $\mathrm{G}_{1}$ (Prakash, 2018), Azospirillum ACD-15 (Sandesh, 2016), Psuedomonas striata (Parvathi and Patil, 2018) and KSB developed at the Institute of Organic Farming (IOF), University of Agricultural Sciences, Dharwad were borrowed and used in this study.

Entrapment of microbial strains within Naalginate beads was carried out under aseptic conditions as proposed by Saxena (2011). Sodium alginate powder and $0.1 \mathrm{M} \mathrm{CaCl}_{2}$ were prepared and autoclaved separately. The population of each inoculant in consortia was set to $10^{8} \mathrm{CFU} / \mathrm{ml}$ and to that sterilized Naalginate powder was added @ 2 g/100 ml separately and thoroughly stirred to obtain a homogenous mixture. Then the mixture was extruded through sterilized burette at steady rate into gently stirred, sterilized $0.1 \mathrm{M} \mathrm{CaCl}_{2}$ solution at room temperature to form uniform sized beads. Thus formed beads were kept in $0.1 \mathrm{M} \mathrm{CaCl}_{2}$ solution at room temperature to obtain regular solid beads and later the $\mathrm{CaCl}_{2}$ solution was drained and the beads were washed twice with sterile distilled water and dried in laminar air flow for $12 \mathrm{hr}$.

\section{In vitro study on maize}

Clean, bold and healthy maize (Super 900M Gold) seeds were surface sterilized with alcohol $(70 \%)$ for one minute, followed by sodium hypochlorite ( $2 \%$ active chlorine) for three minutes. These treated seeds were then washed six times with the sterile distilled water and were allowed to dry under a laminar air flow chamber then treated with the developed formulations of microbial consortia. The surface sterilized, dried seeds 
were treated with carrier based NPK consortia (lignite, kaolinite and bentonite) @ $20 \mathrm{~g} \mathrm{~kg}^{-1}$ of seeds and liquid consortia@ $4 \mathrm{ml} \mathrm{kg}^{-1}$ and Na-alginate consortia dissolved in small quantity of sterile distilled water @ $4 \mathrm{~g} \mathrm{~kg}^{-1}$ of seeds. Recommended dose of lignite based Azospirillum ACD-15 and PSB @ $20 \mathrm{~g} \mathrm{~kg}^{-1}$ was used as a check. The germination test was conducted by following the procedure given by ISTA (1999) using between paper method.

\section{Shelf life evaluation}

The shelf life of individual biofertilizer strains in each formulation was assessed at 30 days interval for 90 days after incubation by Direct Plate Count Technique (DPCT) for all the four strains and Most Probable Number Estimation (MPNE) method for Gluconacetobacter $\mathrm{G}_{1}$ and Azospirillum ACD-15 correspondingly on semi-solid LGIP and sodium malate medium.

The selective media used were LGIP for Gluconacetobacter, sodium malate medium for Azospirillum, Pikovaskaya's medium for PSB and Alekshandrov's medium for KSB.

\section{Statistical analysis}

The data obtained from in vitro studies were subjected to statistical analysis using Factorial Completely Randomized Design (FCRD) and data on shelf life of formulations of biofertilizer consortia were subjected to statistical analysis using Completely Randomized Design (CRD). Interpretation of the data was carried out in accordance with Panse and Sukhatme (1985).

The level of significance used in the ' $F$ ' and ' $\mathrm{t}$ ' test was $\mathrm{P}<0.01$. The mean values between treatments were compared using the least significance differences (L. S. D). The treatment means were compared by applying Duncan's Multiple Range Test (DMRT).

\section{Results and Discussion}

\section{Compatibility of biofertilizer strains}

An in vitro experiment was conducted to test the compatibility of the biofertilizer strains used in this study. The four local efficient microbial strains viz., Gluconacetobacter $\mathrm{G}_{1}$, Azospirillum ACD-15, Psuedomonas striata (PSB) and a KSB were found compatible with each other as evident from a cross streak assay on a common growth medium (Nutrient agar). This ensured no variation in formulations developed using the four biofertilizer strains was due to competition among them. Secondly, physical mixing of the strains did not have any deterrent effect on the performance of each other in the final consortia. The results also demonstrated the significance of conducting compatibility test among constituent biofertilizer strains following a simple procedure. If carefully followed, this test can reveal compatibility or competition arising due to physical competition and antibiosis as revealed on nutrient agar plates and is prerequisite for developing consortia.

\section{Shelf life of formulations}

Before mixing for developing formulations, the populations $(\mathrm{CFU} / \mathrm{ml})$ of strains viz., Gluconacetobacter $\mathrm{G}_{1}$, Azospirillum ACD-15, PSB and KSB on their respective selective medium were $7.43 \times 10^{10}, 38.6 \times 10^{8}, 34 \times 10^{9}$ and $23 \times 10^{8}$ respectively. Among them KSB had the least population. As consortia were developed based on equal population of each biofertilizer strain volume of each culture with different population required was calculated. One $\mathrm{ml}$ of KSB culture containing $23 \times 10^{8} \mathrm{CFU} / \mathrm{ml}$ was equivalent in population to $0.03 \mathrm{ml}$ of Gluconacetobacter $\mathrm{G}_{1}$ containing $7.43 \times 10^{10} \mathrm{CFU} / \mathrm{ml}, 0.06 \mathrm{ml}$ of PSB containing $34 \times 10^{8} \mathrm{CFU} / \mathrm{ml}$ and $0.59 \mathrm{ml}$ of Azospirillum ACD-15 containing $38.60 \times 10^{8} \mathrm{CFU} / \mathrm{ml}$. 
Table.1 Time taken by biofertilizer strains to reach maximum population

\begin{tabular}{|l|c|c|c|}
\hline Biofertilizer strains & $\begin{array}{c}\text { Time } \\
\text { (hr) }\end{array}$ & $\begin{array}{c}\text { Maximum population } \\
\text { (media without cell } \\
\text { protectants) }\end{array}$ & $\begin{array}{c}\text { Maximum population } \\
\text { (media with cell } \\
\text { protectants-for Liquid } \\
\text { formulation) }\end{array}$ \\
\hline Gluconacetobacter G & 96 & $7.43 \times 10^{10}$ & $12.56 \times 10^{10}$ \\
\hline Azospirillum ACD-15 & 56 & $38.6 \times 10^{8}$ & $125.6 \times 10^{9}$ \\
\hline $\begin{array}{l}\text { Pseudomonas striata } \\
\text { (PSB) }\end{array}$ & 48 & $34 \times 10^{9}$ & $57 \times 10^{9}$ \\
\hline KSB & 36 & $23 \times 10^{8}$ & $32 \times 10^{8}$ \\
\hline
\end{tabular}

Table .2 Additives, adjuvant, surfactants, antioxidant and their concentrations used in developing liquid formulations of microbial consortia

\begin{tabular}{|c|c|c|c|c|c|}
\hline \multirow{2}{*}{\multicolumn{2}{|c|}{ Amendments }} & \multicolumn{4}{|c|}{ Concentrations of amendments } \\
\hline & & $\mathbf{G}_{1}$ & ACD-15 & PSB & KSB \\
\hline \multirow[t]{3}{*}{ Additives } & Polyethylene Glycol (PEG) (\%) & 0.5 & 0.5 & 0.5 & 0.5 \\
\hline & Trehalose (mM) & 5 & 5 & - & - \\
\hline & Glycerol (mM) & 5 & 5 & 5 & 5 \\
\hline Adjuvant & Gum Arabica (\%) & 0.15 & 0.15 & 0.15 & 0.15 \\
\hline Surfactant & Polysorbate-20 (ppm) & & 250 & 250 & 250 \\
\hline Antioxidant & Ascorbic acid & 0.02 & - & - & - \\
\hline
\end{tabular}

Table.3 Proportion of the three microbial inoculants in formulation of two different microbial consortia in $1: 1: 1$ ratio

\begin{tabular}{|c|c|c|c|c|c|c|}
\hline \multirow[t]{2}{*}{$\begin{array}{l}\text { Sl. } \\
\text { No. }\end{array}$} & \multirow[t]{2}{*}{$\begin{array}{l}\text { Microbial } \\
\text { consortia }\end{array}$} & \multirow[t]{2}{*}{$\begin{array}{l}\text { Different } \\
\text { formulations } \\
\text { developed }\end{array}$} & \multicolumn{3}{|c|}{$\begin{array}{l}\text { Volume of microbial } \\
\text { inoculants used in developing } \\
\text { microbial consortia }(\mathrm{ml})\end{array}$} & \multirow{2}{*}{$\begin{array}{l}\text { Final } \\
\text { volume of } \\
\text { microbial } \\
\text { consortia } \\
(\mathrm{ml})\end{array}$} \\
\hline & & & G1 & PSB & KSB & \\
\hline \multirow[t]{2}{*}{1} & \multirow[t]{2}{*}{ MC-1 } & $\begin{array}{l}\text { Lignite } \\
\text { Kaolinite } \\
\text { Bentonite } \\
\text { Na-alginate }\end{array}$ & 1.83 & 4.24 & 60.61 & 66.68 \\
\hline & & Liquid & 4.63 & 10.37 & 185.18 & 200.18 \\
\hline \multirow[t]{2}{*}{2} & \multirow[t]{2}{*}{ MC-2 } & $\begin{array}{l}\text { Lignite } \\
\text { Kaolinite } \\
\text { Bentonite } \\
\text { Na-alginate }\end{array}$ & 24.09 & 2.81 & 40.16 & 67.06 \\
\hline & & Liquid & 4.63 & 10.37 & 185.18 & 200.18 \\
\hline
\end{tabular}


Table.4 Population of Gluconacetobacter strain, $\mathrm{G}_{1}$ in different formulations of microbial consortium- 1 at different interval under ambient condition

\begin{tabular}{|c|c|c|c|c|}
\hline \multirow[t]{2}{*}{ Formulations } & \multicolumn{4}{|c|}{$\begin{array}{c}\text { Population } \mathrm{G}_{\mathbf{1}}\left(\begin{array}{c}\left.\times 10^{8} \mathrm{CFU} / \mathrm{ml}\right) \text { at regular } \\
\text { intervals }\end{array}\right. \\
\text { Days after incubation (DAI) }\end{array}$} \\
\hline & 0 & 30 & 60 & 90 \\
\hline Lignite & $\begin{array}{c}9.700^{c} \\
(50.167)\end{array}$ & $\begin{array}{c}9.072^{\mathrm{b}} \\
(12.000)\end{array}$ & $\begin{array}{l}8.100^{\mathrm{a}} \\
(1.333)\end{array}$ & $\begin{array}{c}7.20^{\mathrm{a}} \\
(0.167)\end{array}$ \\
\hline Kaolinite & $\begin{array}{c}9.729^{c} \\
(53.833)\end{array}$ & $\begin{array}{c}9.033^{\mathrm{b}} \\
(11.167)\end{array}$ & $\begin{array}{c}8.059^{\mathrm{a}} \\
(1.167)\end{array}$ & $\begin{array}{l}7.318^{\mathrm{a}} \\
(0.217)\end{array}$ \\
\hline Liquid & $\begin{array}{c}10.049^{\mathrm{a}} \\
(112.000)\end{array}$ & $\begin{array}{c}9.438^{\mathrm{a}} \\
(27.500)\end{array}$ & $\begin{array}{c}8.159^{\mathrm{a}} \\
(1.500)\end{array}$ & $\begin{array}{l}7.812^{\mathrm{a}} \\
(0.650)\end{array}$ \\
\hline Na-alginate & $\begin{array}{c}9.868^{\mathrm{b}} \\
(74.000)\end{array}$ & $\begin{array}{c}9.704^{\mathrm{a}} \\
(52.667)\end{array}$ & $\begin{array}{c}8.100^{\mathrm{a}} \\
(1.333)\end{array}$ & $\begin{array}{l}7.693^{\mathrm{a}} \\
(0.500)\end{array}$ \\
\hline Bentonite & $\begin{array}{c}9.673^{c} \\
(47.333)\end{array}$ & $\begin{array}{l}8.933^{b} \\
(8.833)\end{array}$ & $\begin{array}{l}8.100^{\mathrm{a}} \\
(1.333)\end{array}$ & $\begin{array}{l}7.301^{\mathrm{a}} \\
(0.233)\end{array}$ \\
\hline S.E.m ( \pm$)$ & 0.022 & 0.069 & 0.091 & 0.101 \\
\hline L.S.D $(p \leq 0.01)$ & 0.101 & 0.308 & NS & 0.452 \\
\hline
\end{tabular}

Note: The values in parenthesis are real values next to the $\log _{10}$ transformed values and statistical analysis was done to $\log _{10}$ transformed values.

Means followed by the same superscript within factors $(A$ and $B)$ and their interaction $(A \times B)$ do not vary significantly at $\mathrm{P}<0.01$ by DMRT.

Table.5 Population of PSB in different formulations of microbial consortium-1 at different interval under ambient condition

\begin{tabular}{|c|c|c|c|c|}
\hline \multirow[t]{3}{*}{ Formulations } & \multirow{2}{*}{\multicolumn{4}{|c|}{$\begin{array}{c}\text { Population PSB }\left(\times 10^{8} \mathrm{CFU} / \mathrm{ml}\right) \text { at regular } \\
\text { intervals } \\
\text { Days after incubation (DAI) }\end{array}$}} \\
\hline & & & & \\
\hline & $\mathbf{0}$ & 30 & 60 & 90 \\
\hline Lignite & $\begin{array}{c}9.602^{\mathrm{b}} \\
(40.000)\end{array}$ & $\begin{array}{l}8.735^{\mathrm{a}} \\
(5.500)\end{array}$ & $\begin{array}{l}8.059^{b} \\
(1.500)\end{array}$ & $\begin{array}{c}7.059^{\mathrm{a}} \\
(0.117)\end{array}$ \\
\hline Kaolinite & $\begin{array}{c}9.618^{\mathrm{b}} \\
(41.500)\end{array}$ & $\begin{array}{l}8.522^{\mathrm{a}} \\
(3.333)\end{array}$ & $\begin{array}{l}8.259^{\mathrm{c}} \\
(1.667)\end{array}$ & $\begin{array}{l}7.259^{\mathrm{a}} \\
(0.200)\end{array}$ \\
\hline Liquid & $\begin{array}{c}9.828^{\mathrm{a}} \\
(68.667)\end{array}$ & $\begin{array}{l}8.583^{\mathrm{a}} \\
(3.833)\end{array}$ & $\begin{array}{l}8.577^{\mathrm{a}} \\
(2.167)\end{array}$ & $\begin{array}{l}7.577^{\mathrm{a}} \\
(0.417)\end{array}$ \\
\hline Na-alginate & $\begin{array}{c}9.699^{\mathrm{ab}} \\
(50.333)\end{array}$ & $\begin{array}{c}8.573^{\mathrm{a}} \\
(4.500)\end{array}$ & $\begin{array}{l}8.360^{\mathrm{ab}} \\
(1.667)\end{array}$ & $\begin{array}{l}7.360^{\mathrm{a}} \\
(0.233)\end{array}$ \\
\hline Bentonite & $\begin{array}{c}9.651^{\mathrm{b}} \\
(44.833)\end{array}$ & $\begin{array}{l}8.059^{b} \\
(1.167)\end{array}$ & $\begin{array}{l}8.259^{a b} \\
(1.500)\end{array}$ & $\begin{array}{l}7.259^{\mathrm{a}} \\
(0.200)\end{array}$ \\
\hline S. $\operatorname{Em}( \pm)$ & 0.033 & 0.098 & 0.115 & 0.115 \\
\hline L.S.D $(p \leq 0.01)$ & 0.147 & 0.438 & NS & NS \\
\hline
\end{tabular}

Note: The values in parenthesis are real values next to the $\log _{10}$ transformed values and statistical analysis was done to $\log _{10}$ transformed values.

Means followed by the same superscript within factors $(\mathrm{A}$ and $\mathrm{B})$ and their interaction $(\mathrm{A} \times \mathrm{B})$ do not vary significantly at $\mathrm{P}<0.01$ by DMRT. 
Table.6 Population of KSB in different formulations of microbial consortium-1 at different interval under ambient condition

\begin{tabular}{|c|c|c|c|c|}
\hline \multirow[t]{2}{*}{ Formulations } & \multicolumn{4}{|c|}{$\begin{array}{c}\text { Population KSB }\left(\times 1^{8} \mathrm{CFU} / \mathrm{ml}\right) \text { at } \\
\text { regular intervals } \\
\text { Days after incubation (DAI) }\end{array}$} \\
\hline & 0 & 30 & 60 & 90 \\
\hline Lignite & $\begin{array}{c}9.561^{\mathrm{b}} \\
(36.833)\end{array}$ & $\begin{array}{c}8.218^{\mathrm{c}} \\
(1.667)\end{array}$ & $\begin{array}{c}8.000^{\mathrm{a}} \\
(1.000)\end{array}$ & $\begin{array}{c}7.133^{\mathrm{a}} \\
(0.150)\end{array}$ \\
\hline Kaolinite & $\begin{array}{c}9.618^{\mathrm{b}} \\
(41.500)\end{array}$ & $\begin{array}{c}8.788^{\mathrm{a}} \\
(6.167)\end{array}$ & $\begin{array}{c}8.159^{\mathrm{a}} \\
(1.500)\end{array}$ & $\begin{array}{c}7.059^{\mathrm{a}} \\
(0.117)\end{array}$ \\
\hline Liquid & $\begin{array}{c}9.705^{\mathrm{a}} \\
(50.667)\end{array}$ & $\begin{array}{c}8.774^{\mathrm{a}} \\
(6.000)\end{array}$ & $\begin{array}{l}8.100^{\mathrm{a}} \\
(1.333)\end{array}$ & $\begin{array}{c}7.300^{\mathrm{a}} \\
(0.200)\end{array}$ \\
\hline Na-alginate & $\begin{array}{c}9.777^{\mathrm{a}} \\
(59.833)\end{array}$ & $\begin{array}{c}8.558^{b} \\
(3.667)\end{array}$ & $\begin{array}{c}8.059^{\mathrm{a}} \\
(1.167)\end{array}$ & $\begin{array}{c}7.218^{a} \\
(0.183)\end{array}$ \\
\hline Bentonite & $\begin{array}{c}9.607^{b} \\
(40.500)\end{array}$ & $\begin{array}{c}8.799^{\mathrm{a}} \\
(6.333)\end{array}$ & $\begin{array}{c}8.100^{\mathrm{a}} \\
(1.333)\end{array}$ & $\begin{array}{c}7.100^{\mathrm{a}} \\
(0.133)\end{array}$ \\
\hline S. $\operatorname{Em}( \pm)$ & 0.022 & 0.040 & 0.079 & 0.101 \\
\hline L.S.D $(p \leq 0.01)$ & 0.098 & 0.180 & NS & NS \\
\hline
\end{tabular}

Note: The values in parenthesis are real values next to the $\log _{10}$ transformed values and statistical analysis was done to $\log _{10}$ transformed values.

Means followed by the same superscript within factors (A and B) and their interaction $(A \times B)$ do not vary significantly at $\mathrm{P}<0.01$ by DMRT.

Table.7 Population of Azospirillum strain, ACD-15 in different formulations of microbial consortium-2 at different interval under ambient condition

\begin{tabular}{|c|c|c|c|c|}
\hline \multirow[t]{3}{*}{ Form } & \multirow{2}{*}{\multicolumn{4}{|c|}{$\begin{array}{c}\text { Population ACD-15 }\left(\times 10^{8} \mathrm{CFU} / \mathrm{ml}\right) \text { at } \\
\text { regular intervals } \\
\text { Days after incubation }(\mathrm{DAI})\end{array}$}} \\
\hline & & & & \\
\hline & 0 & 30 & 60 & 90 \\
\hline Lignite & $\begin{array}{c}9.657^{\mathrm{b}} \\
(45.500)\end{array}$ & $\begin{array}{c}9.327^{\mathrm{b}} \\
(21.333)\end{array}$ & $\begin{array}{c}8.059^{\mathrm{a}} \\
(1.167)\end{array}$ & $\begin{array}{l}7.541^{\mathrm{a}} \\
(0.400)\end{array}$ \\
\hline Kaolinite & $\begin{array}{c}9.695^{\mathrm{b}} \\
(49.667)\end{array}$ & $\begin{array}{c}9.415^{\mathrm{b}} \\
(27.000)\end{array}$ & $\begin{array}{c}8.000^{\mathrm{a}} \\
(1.000)\end{array}$ & $\begin{array}{c}7.259^{\mathrm{a}} \\
(0.200)\end{array}$ \\
\hline Liquid & $\begin{array}{c}10.038^{\mathrm{a}} \\
(109.500)\end{array}$ & $\begin{array}{c}9.356^{\mathrm{b}} \\
(22.833)\end{array}$ & $\begin{array}{l}8.259^{\mathrm{a}} \\
(2.000)\end{array}$ & $\begin{array}{c}7.618^{\mathrm{a}} \\
(0.467)\end{array}$ \\
\hline Na-alginate & $\begin{array}{c}9.935^{\mathrm{a}} \\
(86.167)\end{array}$ & $\begin{array}{c}9.630^{\mathrm{a}} \\
(42.667)\end{array}$ & $\begin{array}{l}8.208^{\mathrm{a}} \\
(1.633)\end{array}$ & $\begin{array}{r}7.700^{\mathrm{a}} \\
(0.517)\end{array}$ \\
\hline Bentonite & $\begin{array}{c}9.669^{\mathrm{b}} \\
(47.000)\end{array}$ & $\begin{array}{c}9.191^{\mathrm{b}} \\
(16.000)\end{array}$ & $\begin{array}{l}8.100^{\mathrm{a}} \\
(1.333)\end{array}$ & $\begin{array}{l}7.100^{\mathrm{a}} \\
(0.133)\end{array}$ \\
\hline S. $\operatorname{Em}( \pm)$ & 0.026 & 0.055 & 0.084 & 0.132 \\
\hline L.S.D $(p \leq 0.01$ & 0.114 & 0.246 & NS & NS \\
\hline
\end{tabular}

Note: The values in parenthesis are real values next to the $\log _{10}$ transformed values and statistical analysis was done to $\log _{10}$ transformed values.

Means followed by the same superscript within factors $(A$ and $B)$ and their interaction $(A \times B)$ do not vary significantly at $\mathrm{P}<0.01$ by DMRT. 
Table.8 Population of PSB in different formulations of microbial consortium-2 at different interval under ambient condition

\begin{tabular}{|c|c|c|c|c|}
\hline \multirow[t]{3}{*}{ Formulations } & \multirow{2}{*}{\multicolumn{4}{|c|}{$\begin{array}{c}\text { Population PSB }\left(\times \mathbf{1 0}^{8} \mathrm{CFU} / \mathrm{ml}\right) \text { at regular } \\
\text { intervals } \\
\text { Days after incubation (DAI) }\end{array}$}} \\
\hline & & & & \\
\hline & $\mathbf{0}$ & 30 & 60 & 90 \\
\hline Lignite & $\begin{array}{c}9.607^{b} \\
(40.500)\end{array}$ & $\begin{array}{l}8.597^{b c} \\
(4.167)\end{array}$ & $\begin{array}{c}8.201^{a} \\
(1.333)\end{array}$ & $\begin{array}{c}7.201^{a} \\
(0.167)\end{array}$ \\
\hline Kaolinite & $\begin{array}{c}9.645^{b} \\
(44.500)\end{array}$ & $\begin{array}{l}8.685^{a b} \\
(5.000)\end{array}$ & $\begin{array}{c}8.191^{\mathrm{a}} \\
(1.000)\end{array}$ & $\begin{array}{c}7.191^{\mathrm{a}} \\
(0.167)\end{array}$ \\
\hline Liquid & $\begin{array}{c}9.798^{\mathrm{a}} \\
(63.000)\end{array}$ & $\begin{array}{c}8.678^{a b} \\
(4.8330)\end{array}$ & $\begin{array}{c}8.259^{\mathrm{a}} \\
(1.500)\end{array}$ & $\begin{array}{c}7.259^{a} \\
(0.183)\end{array}$ \\
\hline Na-alginate & $\begin{array}{c}9.751^{\mathrm{ab}} \\
(56.500)\end{array}$ & $\begin{array}{c}9.107^{\mathrm{a}} \\
(13.000)\end{array}$ & $\begin{array}{c}8.259^{a} \\
(2.667)\end{array}$ & $\begin{array}{c}7.259^{a} \\
(0.200)\end{array}$ \\
\hline Bentonite & $\begin{array}{c}9.640^{b} \\
(44.167)\end{array}$ & $\begin{array}{l}8.201^{c} \\
(1.667)\end{array}$ & $\begin{array}{c}8.159^{a} \\
(1.333)\end{array}$ & $\begin{array}{c}7.159^{a} \\
(0.150)\end{array}$ \\
\hline S. Em $( \pm)$ & 0.031 & 0.080 & $0.102^{\mathrm{a}}$ & 0.102 \\
\hline L.S.D $(p \leq 0.01)$ & 0.138 & 0.361 & NS & NS \\
\hline
\end{tabular}

Note: The values in parenthesis are real values next to the $\log _{10}$ transformed values and statistical analysis was done to $\log _{10}$ transformed values.

Means followed by the same superscript within factors (A and B) and their interaction $(A \times B)$ do not vary significantly at $\mathrm{P}<0.01$ by DMRT.

Table.9 Population of KSB in different formulations of microbial consortium-2 at different interval under ambient condition

\begin{tabular}{|c|c|c|c|c|}
\hline \multirow[t]{2}{*}{ Formulations } & \multicolumn{4}{|c|}{$\begin{array}{c}\text { Population KSB }\left(\times 10^{8} \mathrm{CFU} / \mathrm{ml}\right) \text { at regular } \\
\text { intervals } \\
\text { Days after incubation (DAI) }\end{array}$} \\
\hline & 0 & 30 & 60 & 90 \\
\hline Lignite & $\begin{array}{c}9.570^{\mathrm{a}} \\
(37.167)\end{array}$ & $\begin{array}{r}8.869^{\mathrm{a}} \\
(7.500)\end{array}$ & $\begin{array}{c}8.059^{\mathrm{a}} \\
(1.167)\end{array}$ & $\begin{array}{l}7.201^{\mathrm{a}} \\
(0.167)\end{array}$ \\
\hline Kaolinite & $\begin{array}{c}9.576^{\mathrm{a}} \\
(37.667)\end{array}$ & $\begin{array}{l}8.760^{\mathrm{at}} \\
(5.833)\end{array}$ & $\begin{array}{l}8.000^{\mathrm{a}} \\
(1.000)\end{array}$ & $\begin{array}{l}7.259^{\mathrm{a}} \\
(0.200)\end{array}$ \\
\hline Liquid & $\begin{array}{c}9.684^{\mathrm{a}} \\
(48.500)\end{array}$ & $\begin{array}{l}8.651^{b} \\
(4.500)\end{array}$ & $\begin{array}{l}8.100^{\mathrm{a}} \\
(1.333)\end{array}$ & $\begin{array}{l}7.401^{\mathrm{a}} \\
(0.267)\end{array}$ \\
\hline Na-alginate & $\begin{array}{c}9.720^{\mathrm{a}} \\
(53.333)\end{array}$ & $\begin{array}{l}8.724^{\mathrm{at}} \\
(5.333)\end{array}$ & $\begin{array}{c}8.000^{\mathrm{a}} \\
(1.000)\end{array}$ & $\begin{array}{l}7.324^{\mathrm{a}} \\
(0.217)\end{array}$ \\
\hline Bentonite & $\begin{array}{c}9.609^{\mathrm{a}} \\
(41.000)\end{array}$ & $\begin{array}{r}8.883^{\mathrm{a}} \\
(7.667)\end{array}$ & $\begin{array}{c}8.000^{\mathrm{a}} \\
(1.000)\end{array}$ & $\begin{array}{l}7.059^{\mathrm{a}} \\
(0.117)\end{array}$ \\
\hline S. $\operatorname{Em}( \pm)$ & 0.034 & 0.040 & 0.052 & 0.098 \\
\hline L.S.D $(p \leq 0.01)$ & NS & NS & NS & NS \\
\hline
\end{tabular}

Note: The values in parenthesis are real values next to the $\log _{10}$ transformed values and statistical analysis was done to $\log _{10}$ transformed values.

Means followed by the same superscript within factors (A and B) and their interaction $(\mathrm{A} \times \mathrm{B})$ do not vary significantly at $\mathrm{P}<0.01$ by DMRT. 
Table.10 Most Probable Number Estimation of Gluconacetobacter strain, $\mathrm{G}_{1}$ in microbial consortium-1 under ambient condition

\begin{tabular}{|l|c|c|c|c|}
\hline Formulations & \multicolumn{4}{|c|}{$\begin{array}{c}\text { Most probable number at } \times \mathbf{1 0}^{9} \text { at } \\
\text { regular intervals }\end{array}$} \\
\cline { 2 - 5 } & \multicolumn{4}{|c|}{ Days after incubation (DAI) } \\
\hline Lignite & $\mathbf{0}$ & $\mathbf{3 0}$ & $\mathbf{6 0}$ & $\mathbf{9 0}$ \\
\hline & 9.142 & 8.950 & 8.407 & 8.011 \\
& $(1.440)$ & $(0.907)$ & $(0.310)$ & $(0.120)$ \\
\hline Kaolinite & 9.159 & 9.150 & 8.246 & 8.111 \\
& $(1.467)$ & $(1.433)$ & $(0.187)$ & $(0.130)$ \\
\hline Liquid & 9.104 & 9.079 & 8.665 & 8.277 \\
& $(1.300)$ & $(1.240)$ & $(0.700)$ & $(0.203)$ \\
\hline Na-alginate & 9.244 & 9.167 & 8.872 & 8.442 \\
& $(2.000)$ & $(1.500)$ & $(0.933)$ & $(0.320)$ \\
\hline Bentonite & 9.030 & 9.093 & 8.174 & 7.950 \\
& $(1.133)$ & $(1.333)$ & $(0.150)$ & $(0.091)$ \\
\hline S. Em $(\mathbf{\pm})$ & 0.100 & 0.080 & 0.187 & 0.123 \\
\hline L.S.D (p $\leq \mathbf{0 . 0 1 )}$ & NS & NS & NS & NS \\
\hline
\end{tabular}

Note: The values in parenthesis are real values next to the $\log _{10}$ transformed values and statistical analysis was done to $\log _{10}$ transformed values.

Means followed by the same superscript within factors $(A$ and $B)$ and their interaction $(A \times B)$ do not vary significantly at $\mathrm{P}<0.01$ by DMRT.

Table.11 Most Probable Number Estimation of Azospirillum strain, ACD-15 in microbial consortium-2 under ambient condition

\begin{tabular}{|c|c|c|c|c|}
\hline \multirow[t]{2}{*}{ Formulations } & \multicolumn{4}{|c|}{$\begin{array}{c}\text { Most probable number at } \times 10^{9} \text { at regular } \\
\text { intervals } \\
\text { Days after incubation (DAI) }\end{array}$} \\
\hline & 0 & 30 & 60 & 90 \\
\hline Lignite & $\begin{array}{c}9.507 \\
(3.467)\end{array}$ & $\begin{array}{c}9.001 \\
(1.113)\end{array}$ & $\begin{array}{c}8.993 \\
(1.080)\end{array}$ & $\begin{array}{c}8.312 \\
(0.223)\end{array}$ \\
\hline Kaolinite & $\begin{array}{c}9.564 \\
(6.533)\end{array}$ & $\begin{array}{c}9.159 \\
(1.467)\end{array}$ & $\begin{array}{c}8.967 \\
(1.020)\end{array}$ & $\begin{array}{c}8.412 \\
(0.260)\end{array}$ \\
\hline Liquid & $\begin{array}{c}9.437 \\
(3.833)\end{array}$ & $\begin{array}{c}9.049 \\
(1.157)\end{array}$ & $\begin{array}{c}9.093 \\
(1.447)\end{array}$ & $\begin{array}{c}8.599 \\
(0.673)\end{array}$ \\
\hline Na-alginate & $\begin{array}{c}9.177 \\
(1.667)\end{array}$ & $\begin{array}{c}9.116 \\
(1.407)\end{array}$ & $\begin{array}{c}9.044 \\
(1.147)\end{array}$ & $\begin{array}{c}8.484 \\
(0.437)\end{array}$ \\
\hline Bentonite & $\begin{array}{c}9.407 \\
(3.000)\end{array}$ & $\begin{array}{c}8.913 \\
(0.853)\end{array}$ & $\begin{array}{c}8.904 \\
(0.957)\end{array}$ & $\begin{array}{c}7.873 \\
(0.078)\end{array}$ \\
\hline S. Em ( $( \pm)$ & 0.226 & 0.103 & 0.152 & 0.199 \\
\hline L. S. D $(p<0.01)$ & NS & NS & NS & NS \\
\hline
\end{tabular}

Note: The values in parenthesis are real values next to the $\log _{10}$ transformed values and statistical analysis was done to $\log _{10}$ transformed values.

Means followed by the same superscript within factors (A and B) and their interaction $(\mathrm{A} \times \mathrm{B})$ do not vary significantly at $\mathrm{P}<0.01$ by DMRT 
Int.J.Curr.Microbiol.App.Sci (2021) 10(01): 1825-1838

Table.12 Seed germination percentage and seedling vigour index of maize as influenced by inoculation with formulations of microbial consortia

\begin{tabular}{|c|c|c|c|c|c|c|c|c|c|c|c|c|c|c|c|c|}
\hline \multirow[t]{2}{*}{ Formulations } & \multicolumn{8}{|c|}{ Seed germination $(\%)$} & \multicolumn{8}{|c|}{ Seedling vigour index } \\
\hline & $\mathrm{T}_{1}$ & $\mathrm{~T}_{2}$ & $\mathrm{~T}_{3}$ & $\mathrm{~T}_{4}$ & $\mathrm{~T}_{5}$ & $\mathrm{~T}_{6}$ & $\mathrm{~T}_{7}$ & $\begin{array}{l}\text { Mean } \\
\text { (Consorti } \\
\text { a) A }\end{array}$ & $\mathrm{T}_{1}$ & $\mathrm{~T}_{2}$ & $\mathrm{~T}_{3}$ & $\mathrm{~T}_{4}$ & $\mathrm{~T}_{5}$ & $\mathrm{~T}_{6}$ & $\mathrm{~T}_{7}$ & $\begin{array}{l}\text { Mean } \\
\text { (Consort } \\
\text { ia) } A\end{array}$ \\
\hline MC1 & $\begin{array}{c}89.5 \\
0^{\mathrm{e}}\end{array}$ & $\begin{array}{l}91.50 \\
\text { bcd }\end{array}$ & $\underset{c}{92.25^{b}}$ & $\begin{array}{c}95.0 \\
0^{\mathrm{a}}\end{array}$ & $\underset{\mathrm{de}}{90.25}$ & $\begin{array}{c}91.7 \\
5^{b}\end{array}$ & $\begin{array}{c}89.2 \\
5^{c}\end{array}$ & $91.7^{\mathrm{a}}$ & $\begin{array}{c}2552 . \\
33^{\mathrm{f}}\end{array}$ & $\begin{array}{c}2585.6 \\
8^{f}\end{array}$ & $\begin{array}{c}3235.4 \\
7^{d}\end{array}$ & $\begin{array}{c}4102.3 \\
3^{b}\end{array}$ & $\begin{array}{c}2647.8 \\
9^{f}\end{array}$ & $\begin{array}{c}2514.3 \\
6^{c}\end{array}$ & $\begin{array}{c}2135.0 \\
0^{d}\end{array}$ & $3024.74^{b}$ \\
\hline MC2 & $\begin{array}{l}90.5 \\
0^{\text {cde }}\end{array}$ & $\begin{array}{c}90.00 \\
\mathrm{de}\end{array}$ & $96.25^{\mathrm{a}}$ & $\begin{array}{c}93.0 \\
0^{\mathrm{b}}\end{array}$ & $\begin{array}{l}91.00 \\
\text { cde }\end{array}$ & $\begin{array}{c}91.7 \\
5^{b}\end{array}$ & $\begin{array}{c}89.2 \\
5^{c}\end{array}$ & $92.15^{\mathrm{a}}$ & $\begin{array}{c}2613 . \\
83^{f}\end{array}$ & $\begin{array}{c}2686.1 \\
7^{\text {ef }}\end{array}$ & $\begin{array}{c}4266.7 \\
5^{a}\end{array}$ & $\begin{array}{c}3598.5 \\
4^{c}\end{array}$ & $\begin{array}{c}2790.7 \\
1^{\mathrm{e}}\end{array}$ & $\begin{array}{c}2514.3 \\
6^{c}\end{array}$ & $\begin{array}{c}2135.0 \\
0^{d}\end{array}$ & $3191.20^{\mathrm{a}}$ \\
\hline $\begin{array}{c}\text { Mean } \\
\text { (Formulations) } \\
\text { B }\end{array}$ & $\begin{array}{l}90.0 \\
0^{b}\end{array}$ & $\underset{b}{90.75}$ & $94.25^{\mathrm{a}}$ & $\begin{array}{c}94.0 \\
0^{\mathrm{a}}\end{array}$ & $\underset{b}{90.63}$ & $\begin{array}{c}91.7 \\
5^{b}\end{array}$ & $\begin{array}{c}89.2 \\
5^{c}\end{array}$ & & $\begin{array}{c}2583 \\
08^{\mathrm{b}}\end{array}$ & $\begin{array}{c}2635.9 \\
2^{b}\end{array}$ & $\begin{array}{c}3751.1 \\
1^{\mathrm{a}}\end{array}$ & $\begin{array}{c}3850.4 \\
3^{\mathrm{a}}\end{array}$ & $\begin{array}{c}2719.3 \\
0^{\mathrm{b}}\end{array}$ & $\begin{array}{c}2514.3 \\
6^{c}\end{array}$ & $\begin{array}{c}2135.0 \\
0^{d}\end{array}$ & \\
\hline Comparison of & \multicolumn{4}{|c|}{ S. Em ( \pm$)$} & \multicolumn{4}{|c|}{ L. S. D $(p<0.01)$} & \multicolumn{4}{|c|}{ S. Em $( \pm)$} & \multicolumn{4}{|c|}{ L. S. D $(p<0.01)$} \\
\hline A (Consortia) & \multicolumn{4}{|c|}{0.49} & \multicolumn{4}{|c|}{ NS } & \multicolumn{4}{|c|}{36.40} & \multicolumn{4}{|c|}{141.57} \\
\hline $\begin{array}{c}\text { B } \\
\text { (Formulation) }\end{array}$ & \multicolumn{4}{|c|}{0.78} & \multicolumn{4}{|c|}{3.03} & \multicolumn{4}{|c|}{57.56} & \multicolumn{4}{|c|}{223.84} \\
\hline $\begin{array}{c}\text { Interaction } \\
(\mathbf{A} \times \mathbf{B})\end{array}$ & \multicolumn{4}{|c|}{1.10} & \multicolumn{4}{|c|}{ NS } & \multicolumn{4}{|c|}{81.40} & \multicolumn{4}{|c|}{316.55} \\
\hline
\end{tabular}

Note: $\mathbf{M C}_{\mathbf{1}}$ : Microbial consortia-1, $\mathbf{M C}_{\mathbf{2}}$ : Microbial consortia-2, $\mathbf{T}_{\mathbf{1}}$ : Lignite formulation, $\mathbf{T}_{\mathbf{2}}$ : Kaolinite formulation, $\mathbf{T}_{\mathbf{3}}$ : Liquid formulation, $\mathbf{T}_{\mathbf{4}}$ : Na-alginate formulation,

$\mathbf{T}_{5}$ : Bentonite formulation, $\mathbf{T}_{6}$ : Dual inoculation of ACD-15+PSB, $\mathbf{T}_{7}$ : Uninoculated control and NS: Non-significant.

Means followed by the same superscript within factors $(\mathrm{A}$ and $\mathrm{B})$ and their interaction $(\mathrm{A} \times \mathrm{B})$ do not vary significantly at $\mathrm{P}<0.01$ by $\mathrm{DMRT}$. 
Table.13 Shoot and root length of maize seedling as influenced by inoculation with formulations of microbial consortia

\begin{tabular}{|c|c|c|c|c|c|c|c|c|c|c|c|c|c|c|c|c|}
\hline \multirow[t]{2}{*}{ Formulations } & \multicolumn{8}{|c|}{ Shoot length $(\mathrm{cm})$} & \multicolumn{8}{|c|}{ Root length (cm) } \\
\hline & $\mathrm{T}_{1}$ & $\mathrm{~T}_{2}$ & $\mathrm{~T}_{3}$ & $\mathrm{~T}_{4}$ & $\mathrm{~T}_{5}$ & $\mathrm{~T}_{6}$ & $\mathrm{~T}_{7}$ & $\begin{array}{c}\text { Mean } \\
\text { (Consortia) } \\
\text { A }\end{array}$ & $\mathrm{T}_{1}$ & $\mathrm{~T}_{2}$ & $\mathrm{~T}_{3}$ & $\mathrm{~T}_{4}$ & $\mathrm{~T}_{5}$ & $\mathrm{~T}_{6}$ & $\mathrm{~T}_{7}$ & $\begin{array}{c}\text { Mean } \\
\text { (Consortia) } \\
\text { A }\end{array}$ \\
\hline MC1 & $13.04^{\text {ef }}$ & $12.24^{\mathrm{f}}$ & $16.32^{c}$ & $20.59^{a}$ & $13.70^{\mathrm{e}}$ & $12.77^{\mathrm{c}}$ & $11.22^{d}$ & $15.18^{\mathrm{a}}$ & $15.46^{\mathrm{g}}$ & $16.01^{\mathrm{tg}}$ & $18.76^{d}$ & $22.60^{b}$ & $15.64^{\mathrm{tg}}$ & $14.62^{c}$ & $12.70^{d}$ & $17.69^{b}$ \\
\hline MC2 & $12.53^{\mathrm{t}}$ & $12.51^{\mathrm{f}}$ & $20.97^{\mathrm{a}}$ & $18.79^{b}$ & $14.71^{d}$ & $12.77^{\mathrm{c}}$ & $11.22^{d}$ & $15.90^{\mathrm{a}}$ & $16.31^{\mathrm{f}}$ & $17.35^{\mathrm{e}}$ & $23.38^{\mathrm{a}}$ & $19.93^{c}$ & $15.94^{\mathrm{g}}$ & $14.62^{c}$ & $12.70^{\mathrm{d}}$ & $18.58^{\mathrm{a}}$ \\
\hline $\begin{array}{c}\text { Mean } \\
\text { (Formulations) } \\
\text { B }\end{array}$ & $12.78^{c}$ & $12.37^{\mathrm{c}}$ & $18.65^{\mathrm{a}}$ & $19.69^{a}$ & $14.21^{b}$ & $12.77^{\mathrm{c}}$ & $11.22^{d}$ & & $15.88^{b}$ & $16.68^{b}$ & $21.07^{\mathrm{a}}$ & $21.26^{\mathrm{a}}$ & $15.79^{b}$ & $14.62^{c}$ & $12.70^{d}$ & \\
\hline Comparison of & \multicolumn{4}{|c|}{ S. $\operatorname{Em}( \pm)$} & \multicolumn{4}{|c|}{ L. S. D $(p<0.01)$} & \multicolumn{4}{|c|}{ S. $\operatorname{Em}( \pm)$} & \multicolumn{4}{|c|}{ L. S. D $(p<0.01)$} \\
\hline A (Consortia) & \multicolumn{4}{|c|}{0.24} & \multicolumn{4}{|c|}{ NS } & \multicolumn{4}{|c|}{0.19} & \multicolumn{4}{|c|}{0.75} \\
\hline $\begin{array}{c}\text { B } \\
\text { (Formulation) }\end{array}$ & \multicolumn{4}{|c|}{0.38} & \multicolumn{4}{|c|}{1.49} & \multicolumn{4}{|c|}{0.31} & \multicolumn{4}{|c|}{1.19} \\
\hline $\begin{array}{l}\text { Interaction } \\
(\mathbf{A} \times \mathbf{B})\end{array}$ & \multicolumn{4}{|c|}{0.54} & \multicolumn{4}{|c|}{2.11} & \multicolumn{4}{|c|}{0.43} & \multicolumn{4}{|c|}{1.68} \\
\hline
\end{tabular}

Note: $\mathbf{M C}_{\mathbf{1}}$ : Microbial consortia-1, $\mathbf{M C}_{2}$ : Microbial consortia-2, $\mathbf{T}_{\mathbf{1}}$ : Lignite formulation, $\mathbf{T}_{2}:$ Kaolinite formulation, $\mathbf{T}_{3}$ : Liquid formulation, $\mathbf{T}_{\mathbf{4}}:$ Na-alginate formulation, $\mathbf{T}_{5}$ : Bentonite formulation, $\mathbf{T}_{6}$ : Dual inoculation of ACD-15+PSB, $\mathbf{T}_{7}$ : Uninoculated control and NS: Non-significant.

Means followed by the same superscript within factors $(\mathrm{A}$ and $\mathrm{B})$ and their interaction $(\mathrm{A} \times \mathrm{B})$ do not vary significantly at $\mathrm{P}<0.01$ by DMRT 
The population (CFU/ml) of strain Gluconacetobacter $\mathrm{G}_{1}$, Azospirillum ACD-15, PSB and KSB on their respective selective medium amended with protectants (for liquid formulation) were $12.56 \times 10^{10}, 125.6 \times 10^{8}$, $57 \times 10^{9}$ and $32 \times 10^{8} \mathrm{CFU} / \mathrm{ml}$ respectively. Among them KSB had the least population. One $\mathrm{ml}$ inoculum of KSB containing $32 \times 10^{8}$ $\mathrm{CFU} / \mathrm{ml}$ was equivalent in population to 0.03 $\mathrm{ml}$ of Gluconacetobacter $\mathrm{G}_{1}$ containing $12.56 \times 10^{10}, 0.05 \mathrm{ml}$ of PSB containing $57 \times 10^{8}$ and $0.44 \mathrm{ml}$ of Azospirillum ACD-15 containing $73.72 \times 10^{8} \mathrm{CFU} / \mathrm{ml}$.

Among the five formulations, relatively better shelf life of biofertilizer strains in consortium1 (Table 4, 5 and 6) was recorded in liquid formulation where it recorded significantly higher population of $6.5 \times 10^{7} \mathrm{CFU} / \mathrm{ml}$ of Gluconacetobacter $\mathrm{G}_{1}$, PSB $4.2 \times 10^{7} \mathrm{CFU} / \mathrm{ml}$ of PSB and $2.0 \times 10^{7} \mathrm{CFU} / \mathrm{ml} \mathrm{KSB}$ after 90 days after incubation (DAI). Whereas, the population of the biofertilizer strains in consortium-2 (Table 7, 8 and 9) were found significantly higher in Na-alginate formulation with the population of $5.2 \times 10^{7}$ CFU/ml Azospirillum ACD-15, 2.0 $\times 10^{7}$ $\mathrm{CFU} / \mathrm{ml}$ of PSB and $2.6 \times 10^{7} \mathrm{CFU} / \mathrm{ml}$ of $\mathrm{KSB}$ after $90 \mathrm{DAI}$. In the present investigation, it was noticed that, the initial set population of all biofertilizer strains as mentioned earlier was equal and population marginally declined in all the formulations after 90 DAI.

The cell protectants in liquid formulation and encapsulation of biofertilizer strains in $\mathrm{Na}$ alginate formulation played a crucial role in reducing the process of cell desiccation due to their high water holding capacity and thereby maintained water around the cells as compared to other formulations. The main advantages of $\mathrm{Na}$-alginate encapsulation are their slow release of biofertilizer strains in soil, biodegradable and non-toxic in nature besides having long shelf life.
Viable population of microbial strains (Gluconacetobacter $\mathrm{G}_{1}$ and Azospirillum ACD-15) as estimated by MPNE method are presented in table 10 and 11 . It was recorded that there were no significant differences in the population of microbial strains in all the five formulations of two microbial consortia at all stages of incubation. However, the population of Gluconacetobacter $\mathrm{G}_{1}$ in terms of most probable number recorded was higher in Na-alginate formulation upto 90 DAI $\left(1.5 \times 10^{9} \mathrm{CFU} / \mathrm{ml}\right.$ at $30 \mathrm{DAI}, 9.3 \times 10^{8} \mathrm{CFU} / \mathrm{ml}$ at $60 \mathrm{DAI}$ and $3.2 \times 10^{8} \mathrm{CFU} / \mathrm{ml}$ at $90 \mathrm{DAI}$ ) and Azospirillum ACD-15 in kaolinite formulation at $30 \mathrm{DAI}\left(1.4 \times 10^{9} \mathrm{CFU} / \mathrm{ml}\right)$ and in liquid formulation at $60\left(1.4 \times 10^{9} \mathrm{CFU} / \mathrm{ml}\right)$ and $90 \mathrm{DAI}\left(6.7 \times 10^{8} \mathrm{CFU} / \mathrm{ml}\right)$. Therefore, among the five formulations of microbial consortia, liquid formulation of microbial consortium-1 and Na-alginate formulation of microbial consortium-2 supported higher population of each strain until 90 days of storage under ambient conditions. Among the two consortia, the population of PSB and KSB were higher in microbial consortium-1 compared to microbial consortium-2 (Table 5, 7, 8 and 9). However, the population of strains $\mathrm{G}_{1}$ and ACD-15 estimated using Dilution Plate Count Technique (DPCT) were found to be lower than the population values recorded by MPNE method (Table 10 and 11). Amalraj et al., (2016) had reported that liquid and Na-alginate formulation of microbial consortia containing a nitrogen fixer, a PSB and PGPR were found higher for to 60 days of storage at room temperature and these formulations were also found effective.

\section{In vitro study on maize}

Developed formulations were also tested for their effectiveness on maize under in vitro after 90 DAI of shelf life studies. Maize seeds inoculated with NPK consortia in liquid formulation resulted in significantly the highest seed germination $(94.25 \%)$ whereas 
Na-alginate formulation resulted in significantly higher shoot length $(19.69 \mathrm{~cm})$, root length $(21.26 \mathrm{~cm})$ and seedling vigour index (3850.43) irrespective of the type of $\mathrm{N}_{2}$ fixer used as compared to dual inoculation and uninoculated control. These results were in accordance with the earlier studies with Azospirillum and Phosphobacterium (Mariappan et al., 2014) and phosphorus and potassium solubilizing bacteria on maize (Abou and Abdel, 2012). The improved germination and seedling vigour observed in all the treatments over uninoculated control could be attributed to the release of growth promoting substances besides their ability to mobilize three major ( $\mathrm{N}, \mathrm{P}$ and $\mathrm{K}$ ) nutrients by microbial strains in NPK consortia. It is seldom difficult to enhance plant growth by the application of single biofertilizer strain as it can provide only single nutrient element which can be overcome by the application of NPK consortia which provide all the three major nutrients by multiple microbial mechanisms and also other benefits from different biofertilizer strains in consortia on plant growth. From this in vitro study on maize seedling vigour and also shelf life studies, liquid and $\mathrm{Na}$-alginate formulations of microbial consortia were found promising.

In this study, biofertilizer consortia with $\mathrm{Na}$ alginate and liquid formulations proved effective with respect to population stability of individual strain and their effectiveness on maize seedling vigour irrespective of microbial consortium used. This suggested the importance of carrier material in effectiveness of a microbial consortium as the constituent biofertilizer strains in each consortium remained the same but the carrier material used were different. The findings are useful for developing biofertilizer consortia consisting of a nitrogen fixer, a P-solubilizing bacterium and $\mathrm{K}$-solubilizing bacterium formulated in liquid and $\mathrm{Na}$-alginate entrapment.

\section{Acknowledgement}

Authors are grateful to the Department of Agricultural Microbiology and Institute of Organic Farming (IOF), University of Agricultural Sciences, Dharwad-580005 for providing facilities to carry out this research.

\section{References}

Abou, I. I. and Abdel, M. A., 2012. Impact of rock materials and bio-fertilization on $\mathrm{P}$ and $\mathrm{K}$ availability for maize (Zea Mays) under calcareous soil conditions. Saudi J. Biol. Sci., 19: 55-63.

Alagawadi, A. R. and Gaur, A. G., 1988. Interaction between Azospirillum brasiliense and phosphate solubilizing bacteria and their influence on yield and nutrient uptake of sorghum. Appl. Environ. Microbiol., 143: 637-643.

Amalraj, E. L. D., Mohanty, D., Kumar, G. P., Desai, S., Ahmed, S. M. H., Pradhan, R. and Khan, S.S., 2015. Potential microbial consortium for plant growth promotion of sunflower (Helianthus annuus L.). Proc. Natl. Acad. Sci., India, Sect. B. Biol. Sci., 85(2): 635-642.

Anandaraj, B., Leema, R. and Delapierre, A., 2010. Studies on influence of bioinoculants Pseudomonas fluorescens, Rhizobium sp., Bacillus megaterium in green gram. J. Biosci. Technol., 1(2): 95-99.

Desai, S., Praveen, K. G., Sultana, U., Pinisetty, S., Mir Hassan, A. S. K., Amalraj, E. L. D. and Reddy, G., (2013). Potential microbial candidate strains for management of nutrient requirements of crops. African J. Microbiol. Res., 6(17): 3924-3931.

Jain, A., Singh, S., Sharma, B. K. and Singh, H. B., 2012, Microbial consortium mediated reprogramming of defence 
network in pea to enhance tolerance against Sclerotina sclerotiorum. J. Appl. Microbiol., 112: 537-550.

Jayashree, C. and Jagadeesh, K. S., 2017. Testing the effect of the microbial consortium on growth of vegetable seedlings in a farmer's nursery. Int. J. Microbiol. App. Sci., 6(2): 1636-1369.

Mariappan, N., Srimathi, P. and Sundaramoorthi, L., 2014. Effect of liquid biofertilizers on the enhancement of germination in stored seeds of Pongamia pinnata. J. Agril Crop Res., 2 (11): 218-221.

Panse, V. G. and Sukhatme, P. V., 1985. Statistical Methods for Agricultural Workers, 4th Ed., ICAR, New Delhi (India), pp. 347.

Parvathi, S. and Patil, C. R., 2018. Growth of Pseudomonas striata in liquid formulations as influenced by different concentration of additives, adjuvants ad surfactants. J. Farm Sci., 31(1):74-78.

Prakash, A., 2018. Studies on developing liquid formulations of native isolate of Gluconacetobacter. M. Sc. (Agri.). Thesis, Univ. Agric. Sci., Dharwad,
Karnataka (India).

Raja, P., Uma, S., Gopal, H. and Govindarajan, K., 2006, Impact of bio inoculants on rice root exudates, biological nitrogen fixation and plant growth. J. Biol. Sci., 6(5): 815-823.

Sandesh, P., 2017. Optimization of cell protectants, adjuvant and additives to develop liquid formulation of Azospirillum. M. Sc. (Agri.). Thesis, Univ. Agric. Sci., Dharwad, Karnataka (India).

Saxena, M. J., Chandra, S. and Nain, L., 2013. Synergistic effect of phosphate solubilizing rhizobacteria and arbuscular mycorrhiza on growth and yield of wheat plants. J. Soil Sci. Plant Nutri., 13(2): 511-525.

Vijaykumar, G., Brahmaprakash, G. P., Krishna, N. L. and Mudalagiriyappa. 2020. Screening of the selected formulations of a microbial consortium for their effectiveness on the growth of finger millet (Eleusine coracana L. Gaertn.). J. Pharmacognosy and Phytochem., 9(2): 01-09.

\section{How to cite this article:}

Ashwini and Patil, C. R. 2021. Studies on NPK Biofertilizer Consortia and their Influence on Maize (Zea mays L.) Seedling Vigour. Int.J.Curr.Microbiol.App.Sci. 10(01): 1825-1838. doi: https://doi.org/10.20546/ijcmas.2021.1001.213 